\title{
BOLD and perfusion changes during epileptic generalised spike wave activity
}

\author{
Khalid Hamandi MRCP ${ }^{1}$, Helmut Laufs $\mathrm{MD}^{1}$, Ulrike Nöth $\mathrm{PhD}^{2,3}$, David W \\ Carmichael $\mathrm{PhD}^{1}$, John S Duncan FRCP ${ }^{1}$, Louis Lemieux $\mathrm{PhD}^{1}$
}

Author affiliations:

1) Department of Clinical and Experimental Epilepsy, UCL Institute of Neurology, Queen Square, London and National Society for Epilepsy, Chalfont St Peter, Buckinghamshire, UK.

2) Wellcome Department of Imaging Neuroscience, UCL Institute of Neurology, Queen Square, London, UK.

3) Clinical and Academic Unit of Sleep and Breathing, National Heart \& Lung Institute, Imperial College, London, UK

Keywords: EEG-fMRI, generalized spike wave activity, ASL-fMRI

Running title: Decreased cortical perfusion during GSW

Corresponding author: Khalid Hamandi, MRI Unit, National Society for Epilepsy, Chalfont St Peter, Buckinghamshire, SL9 0RJ, UK (now at Welsh Epilepsy Unit, University Hospital Wales, Cardiff)

Telephone: +441494601300 Fax:+441494875666 E mail: Hamandik@cardiff.ac.uk

Word count: title 10, Abstract 225, Main Body 3789, Tables 1, Figures 4, References 39 


\begin{abstract}
It is unclear whether neurovascular coupling is maintained during epileptic discharges. Knowing this is important to allow appropriate inferences from functional imaging studies of epileptic activity. Recent blood oxygen level dependent (BOLD) functional MRI (fMRI) studies have demonstrated negative BOLD responses (NBR) in frontal, parietal and posterior cingulate cortices during generalised spike wave activity (GSW). We hypothesized that GSW-related NBR commonly reflect decreased cerebral blood flow (CBF). We measured BOLD and cerebral blood flow responses using simultaneous EEG with BOLD and arterial spin label (ASL) fMRI at 3T. Four patients with epilepsy were studied; two with idiopathic generalized epilepsy (IGE), and two with secondary generalized epilepsy (SGE). We found GSW-related NBR in frontal, parietal and posterior cingulate cortices. We measured the coupling between BOLD and CBF changes during GSW and normal background EEG and found a positive correlation between the simultaneously measured BOLD and CBF throughout the imaged volume. Frontal and thalamic activation were seen in two patients with SGE, concordant with the electroclinical features of their epilepsy. There was striking reproducibility of the GSWassociated BOLD response in subjects previously studied at $1.5 \mathrm{~T}$.

Our results are consistent with preserved neurovascular coupling between BOLD and CBF changes in patients with generalized epilepsy and in particular during GSW activity. Cortical activations appear to reflect areas of discharge generation whilst deactivations reflect changes in conscious resting state activity.
\end{abstract}




\section{Introduction}

It is unclear whether cerebral neurovascular coupling is affected in patients with epilepsy. This is an important issue for interpretation of fMRI investigations in these patients. Characterisation of the cerebral blood flow (CBF) and blood oxygen leveldependent response (BOLD) during epileptic discharges is therefore necessary, and in particular to better understand BOLD decreases. Generalised spike wave activity GSW is the hallmark of scalp EEG during absence (transient loss of consciousness) seizures in idiopathic generalised epilepsy (IGE) and is also seen in secondary generalised epilepsy (SGE). The predominant finding in EEG-correlated functional MRI (EEGfMRI) studies of GSW have been positive BOLD responses in the thalamus and negative BOLD responses (NBR) in areas of association cortex (Archer et al., 2003; Gotman et al., 2005; Hamandi et al., 2006; Laufs et al., 2006; Salek-Haddadi et al., 2003), the latter akin to the "default mode" network of resting brain function (Greicius et al., 2003; Mazoyer et al., 2001; Raichle et al., 2001).

The BOLD response arises from regional changes in deoxyhaemoglobin concentration due mainly to changes in $\mathrm{CBF}$, with an additional effect of alterations in blood volume and oxygen extraction (Ogawa et al., 1990). Evidence from activation studies in animals demonstrates that the positive BOLD response reflects increased synaptic activity (Shmuel et al., 2006). However, the origin of NBR may correspond to either an absolute decrease in cerebral blood flow coupled with a decrease in neuronal activity, or an increase in oxygen consumption without, or in excess of any blood flow increase (abnormal neurovascular coupling), or due to vascular steal (Wade, 2002). 
Evidence in healthy humans suggests that NBR equate to decreased neural activity or “deactivations" (Born et al., 2002; Czisch et al., 2004; Parkes et al., 2004). Recently, Shmuel et al used simultaneous fMRI and intracranial electrophysiological recordings in monkeys to show that NBR reflect absolute below-baseline decreases in neuronal activity, with tight coupling between NBR and neural activity decreases in the visual cortex (Shmuel et al., 2006).

We hypothesized that GSW-related NBR would reflect decreased CBF. To test this we measured BOLD and cerebral blood flow using simultaneous EEG with BOLD and arterial spin label (ASL) fMRI using a 3T MRI scanner.

\section{Materials and Methods}

\section{Patients}

We investigated four patients with frequent GSW. Two patients had SGE and two IGE (one with generalised tonic clonic seizures (GTCS), the other with childhood absence epilepsy (CAE)) (Commission on Classification and Terminology of the International League Against Epilepsy, 1989), see table 1 for patient details. Patients gave written informed consent. The study was approved by the Joint Research Ethics committee of the National Hospital for Neurology and Neurosurgery.

\section{EEG Acquisition and Processing}

32 channels of surface EEG were recorded in the MR scanner using MRI compatible hardware (BrainAmp MRplus, Brainproducts, Munich, Germany; BrainCap MR, Easycap, Herrsching-Breitbrunn, Germany). Foam padding and ear defenders were used to minimize motion and improve patient comfort. Scanner and EEG clocks were synchronized, such that EEG sampling relative to scanner gradient switching was 
constant (Mandelkow et al., 2006) facilitating improved EEG quality after imaging and pulse artefact subtraction (Vision Analyzer, Brain Products) (Allen et al., 1998; Allen et al., 2000). The start and stop of GSW events were visually marked on the EEG according to the fMRI time series (with a temporal resolution of $\sim 0.2$ seconds or $\sim 1 / 16$ TR) for use in the fMRI analysis (general linear model).

\section{MRI acquisition}

Imaging was carried out on a 3T Siemens Allegra head scanner (Siemens, Erlangen, Germany) using a standard head transmit/receive coil. Two thirty minute runs of functional MRI with simultaneous EEG were acquired in each patient: 1) a gradient echo (BOLD sensitive) echoplanar imaging (EPI) time series, whole brain coverage (TR (repetition time) / TE (echo time): $3120 / 40 \mathrm{~ms}, 48$ slices, 500 volumes, $3 \times 3 \times 2 \mathrm{~mm}$, $1 \mathrm{~mm}$ inter slice gap, FOV $19.2 \times 19.2 \mathrm{~mm}^{2}$, matrix $\left.64 \times 64\right)$ which we will refer to as BOLD series, 2) a pulsed arterial spin labelling (PASL) sequence (Q2TIPS) (Luh et al., 1999; Nöth et al., 2006) with the PICORE (proximal inversion with a control for off resonance effects) labelling scheme where arterial spins are labelled proximal to the imaging slices, which we refer to as ASL series. The scanning parameters were: TR 2.3 sec, (time for acquisition of a single slice was $66 \mathrm{~ms}$, the remainder of the TR being the labelling phase), TE 30ms, 6 axial slices (extending superiorly from the top of the corpus callosum), $4 \mathrm{~mm}$ slice thickness, slice gap $0.5 \mathrm{~mm}$ FOV $22.4 \mathrm{~cm}$ x $22.4 \mathrm{~cm}$, matrix 64 x 64 (see (Nöth et al., 2006) for sequence details). A T1-weighted structural scan with an isotropic resolution of $1 \mathrm{~mm}$ using an MDEFT sequence with optimised contrast for gray matter (GM)-white matter (WM) and imaging parameters as described in (Deichmann et al., 2004) was used to obtain GM and WM masks. 


\section{MRI processing}

The SPM2 (http://www.fil.ion.ucl.ac.uk/SPM) software package was used for image pre-processing and analysis. The structural image of each patient was segmented using SPM2 into GM, WM and CSF. In-house software was written in Matlab (www.mathworks.com) for the calculation of perfusion images. BOLD and ASL series were pre-processed separately.

BOLD series: The first five images were discarded to allow for T1 equilibration effects. Images were spatially realigned, normalized to a standard EPI template based on the MNI reference brain (Ashburner and Friston, 1999) and spatially smoothed (8mm full width at half maximum isotropic Gaussian kernel).

ASL series: The Siemens MoCo (motion correction in frequency space before image reconstruction) series of label and control images were used for analysis. Images were further realigned to the first image using SPM2. A time series of the difference images (control minus label) was calculated by subtracting adjacent control and temporally adjusted label images; a 'surround average' of preceding and following label images was used to remove effects of BOLD signal fluctuation within one TR (Aguirre et al., 2002), and expressed as a ratio of the control image to remove BOLD contrast present in both label and control images (Garraux et al., 2005).

We thresholded difference images according to Garraux et al (Garraux et al., 2005). In brief, abnormal flow values that did not fall within a physiological range (e.g. due to head motion) were removed, by retaining only 1) pairs of voxels where control signal intensity had a value greater than $80 \%$ of the global mean intensity of the control 
image, and 2) voxels with a fractional signal change of less than $\pm 5 \%$. We will refer to this ratio difference image as ASL-P (ASL-Perfusion).

A corresponding BOLD sensitive time series was calculated from the ASL data by summation of adjacent label and control images; we will refer to these as ASL-B (ASLBOLD).

A further time series of difference only images (i.e. not expressed as a ratio) was calculated for quantitative analysis. The mean perfusion images, perfusion time series and structural images were spatially co-registered to a respective BOLD image for each subject, to allow masking of quantitative perfusion images with the GM map.

\section{Statistical analysis}

Blocks corresponding to GSW epochs were convolved with a canonical haemodynamic response function and its temporal derivative (Henson, 2003). These were entered into a design matrix along with a Volterra expansion of the 6 realignment parameters yielding 24 confounds (Henson, 2003). A mass univariate approach as implemented in SPM was used for whole brain analysis, or in the case of perfusion, the acquired 6 slices. In the analysis of the BOLD series and the ASL-B images an autocorrelation (AR1) correction and a $128 \mathrm{~Hz}$ low pass filter were applied (Henson, 2003). In the analysis of the ASL-P images no modelling of intrinsic autocorrelation, filtering or temporal smoothing were applied (Wang et al., 2003).

SPM uses an implicit mask during the estimation of statistical correlation, based on the inclusion of voxels with intensities higher than $80 \%$ of the global image mean. Whilst this is appropriate for BOLD analysis allowing inclusion of within brain voxels only, it is unsuitable for ASL data in that within brain voxel values in the subtraction images 
can be lower than those outside. We therefore used an explicit mask from the subjects' mean perfusion image to define voxels to be included in the ASL-P statistical analysis. The brain mask was obtained by segmentation of the mean EPI image using a seed and region-growing method (Lemieux et al., 2000).

T-contrasts for positive and negative HRF were used to map GSW associated changes. The resulting statistical parametric maps, $\operatorname{SPM}\{t\}$, from individual analyses were thresholded at $\mathrm{P}<0.05$ using the family wise error (FWE) correction for multiple comparisons based on Gaussian random field theory (Friston et al., 1991) for the BOLD timeseries, and $P<0.001$ (uncorrected) for the perfusion images given their lower signal to noise ratio (Loring et al., 2002).

\section{Quantitative CBF analysis}

Absolute difference images acquired during periods of normal EEG activity (rest images) were used for the quantitative analysis. Rest images were defined as those acquired beyond 5 TRs (23 seconds) after the end of any given GSW and up to 1 TR before the start of the following GSW. These time values were chosen to avoid inclusion of images with GSW induced perfusion changes that may precede or persist longer than the EEG defined event EEG (Diehl et al., 1998) but still provide a sufficient number of rest images to increase the signal to noise ratio of the mean perfusion image and hence the most reliable quantitative values.

Quantitative perfusion images were calculated using the average white matter signal $\left(\mathrm{S}_{\mathrm{WM}}\right)$ intensity of the control images to normalize signal intensity - taking into account the differences in spin density and relaxation times - to calculate the signal intensity for fully relaxed arterial blood (Nöth et al., 2006). A GM $>0.7$ probability map obtained 
from segmentation of the structural images was used to create a binary GM mask. This was multiplied with the quantitative CBF map resulting in a GM-CBF map and the mean of this map provided an estimate of global GM perfusion.

\section{BOLD and CBF correlation analysis}

To investigate the coupling between the ASL and BOLD measurements, we performed a voxel-wise correlation analysis between the two. This was done on a) the entire time series, b) the rest epochs (images acquired during background EEG activity), and c) the GSW epochs (images acquired during GSW).

Firstly, a design matrix was created using SPM in an identical manner to that described earlier, but omitting the 'effects of interest' (the onset and duration of epochs of GSW) for both the ASL and BOLD time series in order to remove confounding variance (motion, scanner drift). From this a residual time series of images was calculated using the SPMd toolbox (http://www.sph.umich.edu/ nichols/SPMd).

The residual time series for each voxel was then examined using a one way analysis of covariance (ANCOVA) as implemented in the aoctool.m function in the Matlab statistics toolbox (www.mathworks.com). The correlation between BOLD and perfusion time courses was examined, with an additional regressor used to assign the data at each time point to either 1) a rest epoch (images acquired during background EEG activity), or 2) a GSW epoch (images acquired during GSW). The results of this analysis give the correlation coefficients and their significance. In particular, we examined the overall correlation between BOLD and perfusion signals, where a significant correlation at a voxel level was taken as $|\mathrm{r}|>0.3$ and $\mathrm{p}<0.05$ (Bonferroni corrected by the number of voxels within the explicit mask used for the perfusion SPM 
analysis) with voxels not meeting these criteria set to zero (masked). For voxels meeting these criteria, the correlation coefficients were obtained for rest and GSW periods and a significant difference in correlation between periods taken as $\mathrm{p}<0.001$ uncorrected. This lower threshold was used to look for these more subtle changes rather than the Bonfferoni correction, which is conservative, as it considers all voxels to be independent.

To clearly visualize the spatial overlap of the correlation analyses and the BOLD activation an overlay of statistical maps was created. It shows voxels of significant correlation (both between BOLD and perfusion signals, and differences in correlations between GSW and rest), (de-) activation, and overlap in different colours (figure 4).

\section{Results}

Good quality EEG allowed unambiguous identification of GSW epochs. Figure 1 shows a segment of artefact corrected EEG recorded during the BOLD fMRI demonstrating GSW for each of the patients. Patient characteristics and the number, median and total duration of GSW epochs during each acquisition and for the previously acquired $1.5 \mathrm{~T}$ data (Hamandi et al., 2006), and 3T BOLD and ASL runs are shown in table 1 . The interval between the $1.5 \mathrm{~T}$ and $3 \mathrm{~T}$ experiments ranged from 1 to 4 years.

BOLD responses

Figure 2 (and Table 2 web material) shows maps for positive and negative HRF GSWrelated changes, along with the previously published $1.5 \mathrm{~T}$ results (Hamandi et al., 2006). NBR were seen in all patients at $3 \mathrm{~T}$ with good correspondence with the $1.5 \mathrm{~T}$ results. Patient 1 (SGE) at $1.5 \mathrm{~T}$ had widespread NBR involving all lobes and a small 
mesial frontal activation and a left occipital activation. At $3 \mathrm{~T}$ there was a more extensive mesial and bifrontal activation as well as activation in the temporal and parietal lobes and bilateral cerebellum, with smaller areas of NBR in occipital, bifrontal and temporal areas. Patient 2 (SGE) with EEG evidence of left frontal epileptogenicity showed a small area of left frontal activation at $1.5 \mathrm{~T}$ and $3 \mathrm{~T}$, and thalamic activation at 3T. There was NBR in posterior cingulate, bilateral posterior parietal and frontal areas both at 1.5 and 3T. Patient 3 (GTCS) showed NBR in bilateral posterior parietal and medial anterior occipital areas in both sessions, without areas of activation. Patient 4 (CAE) showed a widespread NBR throughout the cortex, with a medial orbito-frontal maximum at 1.5 and $3 \mathrm{~T}$. In the $1.5 \mathrm{~T}$ session and to a greater extent in the $3 \mathrm{~T}$ session there was signal increase in the cerebral ventricles and periventricular areas.

\section{CBF responses}

SPM analysis of the ASL-P and ASL-B series revealed areas of significant perfusion and BOLD change that were similar (figure 3), and consistent with the whole-brain BOLD results, in regions of overlapping coverage (figure 2). Patient 1 showed an increase in perfusion in mesial frontal areas and decreased perfusion in the precuneus. Patient 2 showed decreases in perfusion in posterior parietal, posterior cingulate and frontal areas. In Patient 3, no significant perfusion change was observed. Patient 4 showed widespread decrease in perfusion throughout the scanned part of the cortex involving posterior parietal, posterior cingulate and frontal areas with a frontal maximum.

\section{Quantitative $C B F$}

Quantitative analysis showed a mean resting perfusion across all 6 slices of 56.8 ( \pm

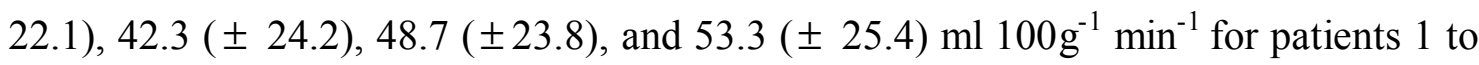


4 respectively. The perfusion decrease at the voxel 'maximum' for patients 1,2 and 4 were $-11.1 \%,-23.5 \%$ and $-37 \%$ respectively.

\section{$B O L D$ and $C B F$ correlation analysis}

Over the whole time series, irrespective of EEG state, nearly the entire imaged brain volume showed a significant positive correlation between BOLD and CBF signal in all cases (figure 4). This held true when epochs of normal background EEG and GSW were studied separately. There was a strong positive correlation between BOLD and CBF in regions that showed NBR (figure 4) comprising all regions of significant bold activation and deactivation. In addition, in regions with significant activation or NBR there were no areas of mismatch (low correlation) between CBF and BOLD responses. Statistical comparison showed a stronger correlation between CBF and BOLD during GSW than at rest; these were co-localised with regions of strongest BOLD (de-) activation. (c.f. dark red regions in the bottom row of statistic maps (figure 4) and spmT maps in figure 4a and bottom 2 rows of figure 4a). Some additional regions of significant change in correlation between rest and GSW were seen (light blue regions in the statistics maps in figure 4). These were generally in regions exhibiting low correlation at rest.

\section{Discussion}

BOLD and CBF were positively correlated in patients with epilepsy during background EEG activity and GSW. This correlation was on average stronger during GSW. We found GSW-related NBR in frontal, parietal and posterior cingulate cortices. Frontal and thalamic activation were seen in two patients with SGE, concordant with the electroclinical features of their epilepsies. Furthermore, striking reproducibility of the GSWassociated BOLD response was observed in subjects previously studied at $1.5 \mathrm{~T}$. 
The finding of GSW-related cortical NBR is consistent with previous studies (Archer et al., 2003; Gotman et al., 2005; Hamandi et al., 2006; Laufs et al., 2006; Salek-Haddadi et al., 2003). The cortical distribution of signal change in frontal, parietal and posterior cingulated cortices, comprise areas of association cortex that are hypothesized to be involved in an organized baseline level of activity (the so-called "default mode'") (Greicius et al., 2003; Mazoyer et al., 2001; Raichle et al., 2001). Our perfusion results and the strong positive correlation between BOLD and perfusion show that these NBR correspond to decreases in cerebral perfusion. In patient 4 NBR were also seen in visual and other cortical areas. Although local cluster maxima overlapped widely with default mode areas described by Raichle et al (Raichle 2003), this suggests more widespread cortical effects of GSW in this case. In addition, more cortical areas might be involved in discharge generation that are not apparent due to possible interference by NBR.

Previously, normal coupling between haemodynamic and metabolic responses was found to be preserved in selected brain regions in two cases with epilepsy (Stefanovic et al., 2005). The changes in cerebral metabolic rate of oxygen (CMRO2), BOLD and CBF were in the normal range, in the selected brain regions (Stefanovic et al., 2005). These regions were selected based on statistical thresholding in relation to a motor paradigm and interictal epileptiform activity in two patients, and the possibility of altered coupling outside, or at the periphery of, these regions was not considered. In both our and their study only a small number of patients were studied in view of the need for sufficiently high GSW events to compensate for the low signal to noise ratio of ASL. 
In our study, we characterised the coupling between BOLD and CBF in cerebral areas affected by GSW (as revealed by the BOLD and CBF maps) and also in unaffected regions. We found a positive correlation between BOLD and perfusion signals across the entire imaging sessions suggesting preserved coupling both during the rest state (background EEG), as well as during epileptic activity, with the latter showing even stronger coupling. There were some local significant differences between the GSW and rest periods at a threshold of $\mathrm{p}<0.001$ uncorrected (see figure 4). These results could indicate regional changes in neurovascular coupling between different states, or nonlinearity in this coupling. However, it is more likely that they reflect areas that show the largest changes in spontaneous activity providing a greater functional contrast range relative to the noise background.

The failure to detect significant perfusion changes in patient 3 was likely due to the low signal to noise ratio of ASL in combination with the low efficiency of this session with only 12 events of 23 seconds total duration, compared to 38 - 68 events (total duration 221 - 564 seconds for the other 3 patients (see table 1). A significant correlation between the BOLD and perfusion signal was found in this patient with a similar pattern to that observed in other subjects.

The mean resting state cerebral perfusion across the whole volume in our subjects was similar to that reported in healthy subjects (Garraux et al., 2005). GSW-related decreases were in the range $11 \%$ to $37 \%$ in broad agreement with neuronally driven deactivations observed during cognitive and motor paradigms (Garraux et al., 2005) and blood flow velocity decreases seen in transcranial Doppler studies of GSW (De Simone et al., 1998; Diehl et al., 1998). In addition to TCD studies, decreases in perfusion have also been 
observed during human absences and GSW, using near infrared spectroscopy (Buchheim et al., 2004) and the ${ }^{133}$ Xenon method (Sperling and Skolnick, 1995).

In animal models the majority of thalamocortical neurons are steadily hyperpolarized and inactive during cortically generated spike wave seizures, with cortical dis-facilitation or 'temporal absence of network activity' (Timofeev and Steriade, 2004). Our findings of preserved neurovascular coupling, are consistent with GSW-related NBR reflecting this decrease in neuronal activity (Shmuel et al., 2006). The extent of the observed NBR in the absence of large activations in some patients makes vascular stealing as a mechanism for NBR (Wade, 2002) highly unlikely. Quantitative perfusion measures were normal and in this context there is little evidence to support the notion of vascular stealing (Born et al., 2002)(Czisch et al., 2004)(Parkes et al., 2004)(Smith et al., 2004).

The positive BOLD changes observed in this study were variable, in line with previous EEG-fMRI studies (Archer et al., 2003; Gotman et al., 2005; Hamandi et al., 2006; Laufs et al., 2006; Salek-Haddadi et al., 2003). Cortical activations concordant with the electroclinical diagnosis were seen in the 2 patients with SGE. In patient 1 , the ictal semiology was typical of prefrontal seizures, suggesting orbitofrontal and anterior cingulate involvement (Niedermeyer, 1998) and concordant with the prefrontal activation. In patient 2, GSW had a left frontal onset in addition to independent left frontal discharges consistent with left frontal and thalamic activations.

In this study, significant thalamic activation was seen in only one patient (\#2). Thalamic fMRI activation has been seen in previous studies (Gotman et al., 2005; Hamandi et al., 2006; Salek-Haddadi et al., 2003) in line with the well documented role of the thalamus in GSW (Avoli et al., 2001; Steriade, 2005). The absence of thalamic activation in the 
other cases may be due to a lack of fMRI sensitivity to detect these changes; Laufs et al found thalamic activation in one case at 3T that was not seen at 1.5T (Laufs et al., 2006). The contrast between the degree of intra-subject similarity between the BOLD maps at $1.5 \mathrm{~T}$ and $3 \mathrm{~T}$ and the degree of inter-subject variability is striking considering the time intervals involved (up to four years), the differences in the number of GSW events between intra-subject sessions and use of different scanners. Given that studies in much

larger groups failed to reveal syndrome-specific patterns (Hamandi et al., 2006) this suggests that the vascular changes are relatively specific in individual patients.

Significant activation was seen in the ventricles in one patient (\#4). This has been previously reported in group analyses of GSW (Gotman et al., 2005; Hamandi et al., 2006). The possible explanations for this effect include GSW-related head motion or altered CSF pulsation.

\section{Conclusion}

In the patients studied here the relationship between BOLD and CBF changes during rest and GSW activity was preserved consistent with normal neurovascular coupling. Cortical activations appeared to reflect areas of discharge generation whilst deactivations reflected decreases in resting state neural activity.

\section{Acknowledgements}

This work was funded by the Wellcome Trust, grant number 067176 (KH, LL), and the Deutsche Forschungsgemeinschaft, grant number LA 1452/3-1 (HL). The National Society for Epilepsy (NSE) supports the MRI Unit. We thank Professor Ray Dolan and O. Josephs (Institute of Neurology, UCL) for their support, and Dr Torben Lund 
(Danish Research Centre for MR) and Professor Robert Turner (Institute of Neurology, UCL) for helpful discussions on the perfusion analysis. 
Table 1. Patient characteristics and details of EEG events during each scan run.

\begin{tabular}{|c|c|c|c|c|c|c|c|c|}
\hline \multirow[t]{4}{*}{ Age } & \multirow[t]{4}{*}{ Sex } & \multirow[t]{4}{*}{ Diagnosis } & Seizures & AEDs & Interictal EEG & \multicolumn{3}{|l|}{ GSW events during fMRI } \\
\hline & & & \multicolumn{3}{|l|}{ Type frequency / (age } & \multicolumn{3}{|c|}{ Number (duration range / median duration / total duration) } \\
\hline & & & onset) & & & (seconds) & & \\
\hline & & & & & & 1.5T BOLD & 3T BOLD & 3T ASL \\
\hline 39 & $\mathrm{~F}$ & SGE & $\begin{array}{l}\operatorname{Abs}^{1} 10-20 / \text { d (9) } \\
\text { GTCS } 0-4 / \text { mnth (11) }\end{array}$ & $\begin{array}{l}\text { CBZ, } \\
\mathrm{LEV}\end{array}$ & $\begin{array}{l}2-3 \mathrm{~Hz} \text { s W with } \\
\text { admixed spikes }\end{array}$ & $57(1.1-25 / 4 / 387)$ & $43(1.6-26 / 7.4 / 387)$ & $\begin{array}{l}38(0.3-8.7 / 6.8 / \\
405)\end{array}$ \\
\hline 21 & M & SGE & $\begin{array}{l}\text { Abs 8/mnth (13) } \\
\text { GTCS 4/yr (18) }\end{array}$ & $\begin{array}{l}\text { CBZ, } \\
\text { LEV,VPA }\end{array}$ & $\begin{array}{l}2-3 \mathrm{~Hz} \text { GSW } \\
\mathrm{L} \text { frontal onset }\end{array}$ & $32(1.1-29 / 5 / 258)$ & $40(0.7-47 / 2.3 / 416)$ & $\begin{array}{l}44(1.7-41.2 / 9.8 \\
/ 564)\end{array}$ \\
\hline 35 & M & EGTCS & GTCS 3 in total (12) & VPA & $3 \mathrm{~Hz}$ GSW & $33(0.6-3.0 / 1.5 / 52)$ & $25(0.8-3.0 / 1.5 / 21)$ & $\begin{array}{l}12(0.7-4.7 / 1.2 / \\
23)\end{array}$ \\
\hline 30 & $\mathrm{~F}$ & CAE & Abs 15/d (8) & None & 3 Hz GSW & $130(0.3-28.7 / 3.3 / 591)$ & $84(1.0-8.3 / 2.6 / 199)$ & $\begin{array}{l}68(0.8-9.8 / 2.8 / \\
221)\end{array}$ \\
\hline
\end{tabular}

Abs - absences, AEDs - anti-epileptic drugs, At Abs - atypical absences, SGE - secondary generalized epilepsy, EGTCS - epilepsy with generalized tonic clonic seizures, CAE - childhood absence epilepsy,. GTCS - generalized tonic-clonic seizures, CBZ - carbamazepine, LEV - levetiracetam, VPA - sodium valproate.

${ }^{1}$ blank spells, on occasion with abnormal posturing (looks left, dystonic posturing of both arms), occasional urinary incontinence, clusters associated with thought disorder (formed illusions, distortion of perception and auditory hallucinations). 
Hamandi et al. BOLD and perfusion changes during GSW 
Reference List

Aguirre, G.K., Detre, J.A., Zarahn, E., Alsop, D.C., 2002. Experimental design and the relative sensitivity of BOLD and perfusion fMRI. NeuroImage 15, 488-500.

Allen, P.J., Josephs, O., Turner, R., 2000. A Method for Removing Imaging Artifact from Continuous EEG Recorded during Functional MRI. NeuroImage 12, 230239.

Allen, P.J., Polizzi, G., Krakow, K., Fish, D.R., Lemieux, L., 1998. Identification of EEG Events in the MR Scanner: The Problem of Pulse Artifact and a Method for Its Subtraction. NeuroImage 8, 229-239.

Archer, J.S., Abbott, D.F., Waites, A.B., Jackson, G.D., 2003. fMRI "deactivation" of the posterior cingulate during generalized spike and wave. NeuroImage 20, 1915-1922.

Ashburner, J., Friston, K.J., 1999. Nonlinear spatial normalization using basis functions. Hum.Brain Mapp. 7, 254-266.

Avoli, M., Rogawski, M.A., Avanzini, G., 2001. Generalized epileptic disorders: an update. Epilepsia 42, 445-457.

Born, A.P., Law, I., Lund, T.E., Rostrup, E., Hanson, L.G., Wildschiodtz, G., Lou, H.C., Paulson, O.B., 2002. Cortical deactivation induced by visual stimulation in human slow-wave sleep. NeuroImage 17, 1325-1335.

Buchheim, K., Obrig, H., Pannwitz, W., Muller, A., Heekeren, H., Villringer, A., Meierkord, H., 2004. Decrease in haemoglobin oxygenation during absence seizures in adult humans. Neurosci.Lett. 354, 119-122.

Commission on Classification and Terminology of the International League Against Epilepsy, 1989. Proposal for revised classification of epilepsies and epileptic syndromes. Commission on Classification and Terminology of the International League Against Epilepsy. Epilepsia 30, 389-399.

Czisch, M., Wehrle, R., Kaufmann, C., Wetter, T.C., Holsboer, F., Pollmacher, T., Auer, D.P., 2004. Functional MRI during sleep: BOLD signal decreases and their electrophysiological correlates. Eur.J Neurosci 20, 566-574.

De Simone, R., Silvestrini, M., Marciani, M.G., Curatolo, P., 1998. Changes in cerebral blood flow velocities during childhood absence seizures. Pediatr.Neurol 18, 132-135.

Deichmann, R., Schwarzbauer, C., Turner, R., 2004. Optimisation of the 3D MDEFT sequence for anatomical brain imaging: technical implications at 1.5 and $3 \mathrm{~T}$. NeuroImage 21, 757-767. 
Diehl, B., Knecht, S., Deppe, M., Young, C., Stodieck, S.R., 1998. Cerebral hemodynamic response to generalized spike-wave discharges. Epilepsia 39, 1284-1289.

Friston, K.J., Frith, C.D., Liddle, P.F., Frackowiak, R.S., 1991. Comparing functional (PET) images: the assessment of significant change. J Cereb Blood Flow Metab 11, 690-699.

Garraux, G., Hallett, M., Talagala, S.L., 2005. CASL fMRI of subcortico-cortical perfusion changes during memory-guided finger sequences. NeuroImage 25, 122-132.

Gotman, J., Grova, C., Bagshaw, A., Kobayashi, E., Aghakhani, Y., Dubeau, F., 2005. Generalized epileptic discharges show thalamocortical activation and suspension of the default state of the brain. Proc Natl Acad Sci 102, 1523615240.

Greicius, M.D., Krasnow, B., Reiss, A.L., Menon, V., 2003. Functional connectivity in the resting brain: a network analysis of the default mode hypothesis. Proc Natl Acad Sci 100, 253-258.

Hamandi, K., Salek-Haddadi, A., Laufs, H., Liston, A., Friston, K., Fish, D.R., Duncan, J.S., Lemieux, L., 2006. EEG-fMRI of idiopathic and secondarily generalized epilepsies. NeuroImage 31, 1700-1710.

Henson, R., 2003. Analysis of fMRI time series. In: Frackowiak, R. S., Friston, K., Frith, C.D., Dolan, R.J., Price, C., Zeki, S., Ashburner, J., Penny, W.D. (Eds.), Human Brain Function, 2nd edition ed. Academic Press.

Laufs, H., Lengler, U., Hamandi, K., Kleinschmidt, A., Krakow, K., 2006. Linking generalized spike-and-wave discharges and resting state brain activity by using EEG/fMRI in a patient with absence seizures. Epilepsia 47, 444-448.

Lemieux, L., Liu, R.S., Duncan, J.S., 2000. Hippocampal and cerebellar volumetry in serially acquired MRI volume scans. Magn Reson.Imaging 18, 1027-1033.

Loring, D.W., Meador, K.J., Allison, J.D., Pillai, J.J., Lavin, T., Lee, G.P., Balan, A., Dave, V., 2002. Now you see it, now you don't: statistical and methodological considerations in fMRI. Epilepsy Behav. 3, 539-547.

Luh, W.M., Wong, E.C., Bandettini, P.A., Hyde, J.S., 1999. QUIPSS II with thin-slice TI1 periodic saturation: a method for improving accuracy of quantitative perfusion imaging using pulsed arterial spin labeling. Magn Reson.Med. 41, 1246-1254.

Mandelkow, H., Halder, P., Boesiger, P., Brandeis, D., 2006. Synchronization facilitates removal of MRI artefacts from concurrent EEG recordings and increases usable bandwidth. Neuroimage. 32, 1120-1126. 
Mazoyer, B., Zago, L., Mellet, E., Bricogne, S., Etard, O., Houde, O., Crivello, F., Joliot, M., Petit, L., Tzourio-Mazoyer, N., 2001. Cortical networks for working memory and executive functions sustain the conscious resting state in man. Brain Res.Bull. 54, 287-298.

Niedermeyer, E., 1998. Frontal lobe epilepsy: the next frontier. Clin.Electroencephalogr. 29, 163-169.

Nöth, U., Meadows, G.E., Kotagima, R., Deichmann, R., Corfield, D.R., Turner, R., 2006. Cerebral vascular response to hypercapnia: determination with perfusion MRI at 1.5 and 3.0 Tesla using a pulsed arterial spin labelling technique. (in press; DOI 10.1002/jmri.20761) ed.

Ogawa, S., Lee, T.M., Kay, A.R., Tank, D.W., 1990. Brain magnetic resonance imaging with contrast dependent on blood oxygenation. Proc Natl Acad Sci 87, 9868-9872.

Parkes, L.M., Fries, P., Kerskens, C.M., Norris, D.G., 2004. Reduced BOLD response to periodic visual stimulation. NeuroImage 21, 236-243.

Raichle, M.E., MacLeod, A.M., Snyder, A.Z., Powers, W.J., Gusnard, D.A., Shulman, G.L., 2001. A default mode of brain function. Proc Natl Acad Sci 98, 676-682.

Salek-Haddadi, A., Lemieux, L., Merschhemke, M., Friston, K.J., Duncan, J.S., Fish, D.R., 2003. Functional magnetic resonance imaging of human absence seizures. Ann Neurol 53, 663-667.

Shmuel, A., Augath, M., Oeltermann, A., Logothetis, N.K., 2006. Negative functional MRI response correlates with decreases in neuronal activity in monkey visual area V1. Nat.Neurosci. 9, 569-577.

Smith, A.T., Williams, A.L., Singh, K.D., 2004. Negative BOLD in the visual cortex: evidence against blood stealing. Hum.Brain Mapp. 21, 213-220.

Sperling, M.R., Skolnick, B.E., 1995. Cerebral blood flow during spike-wave discharges. Epilepsia 36, 156-163.

Stefanovic, B., Warnking, J.M., Kobayashi, E., Bagshaw, A.P., Hawco, C., Dubeau, F., Gotman, J., Pike, G.B., 2005. Hemodynamic and metabolic responses to activation, deactivation and epileptic discharges. NeuroImage 28, 205-215.

Steriade, M., 2005. Sleep, epilepsy and thalamic reticular inhibitory neurons. Trends Neurosci. 28, 317-324.

Timofeev, I., Steriade, M., 2004. Neocortical seizures: initiation, development and cessation. Neuroscience 123, 299-336.

Wade, A.R., 2002. The negative BOLD signal unmasked. Neuron 36, 993-995. 
Wang, J., Aguirre, G.K., Kimberg, D.Y., Roc, A.C., Li, L., Detre, J.A., 2003. Arterial spin labeling perfusion fMRI with very low task frequency. Magn Reson.Med. 49, 796-802. 


\section{Figure 1}

Representative EEG segments from each of the 4 experiments acquired during BOLD EPI at 3T, showing runs of generalized spike wave (GSW). Note the activity in patient \#1 was predominantly slow wave with intermixed spikes and in patient \#2 there is a left frontal lead into the GSW. SGE - secondary generalized epilepsy, GTCS - generalized tonic clonic seizures, CAE - childhood absence epilepsy.

\section{Figure 2}

SPM $\{\mathrm{t}\}$ of BOLD time series for all patients. GSW-related positive (red) and negative (blue) BOLD changes at 1.5T (Hamandi et al., 2006) and 3T overlaid onto the SPM 'T1 template', with 'glass brain' view below. In the overlays, sections were adjusted such that a similar slice orientation is displayed at 1.5 and $3 \mathrm{~T}$ to facilitate comparison. All analyses are thresholded $\mathrm{p}<0.05$ family wise error correction.

SGE-secondary generalized epilepsy, GTCS - generalized tonic clonic seizures, CAE childhood absence epilepsy

\section{Figure 3}

A conjunction of SPM $\{t\}$ from ASL-P and ASL-B time series with positive (BOLD-red and Perfusion-green) and negative (BOLD-blue and Perfusion-cyan), for each of patients \#1, \#2 and \#4, overlaid onto the subjects' mean EPI. The extent and location of the six imaged slices relative to the whole brain can be seen from these activation maps. Comparison with figure 2 shows close correspondence to the whole brain BOLD maps. No significant CBF change was revealed in patient \#3 likely due to the low number of GSW events during the scan run. SGE-secondary generalized epilepsy, CAE-childhood absence epilepsy. 


\section{Figure 4}

a) BOLD-perfusion correlation maps and ASL-B SPM for patient \#4. The central 4 slices (from 6) are displayed (similar results are seen in all slices). Top row: BOLDperfusion correlation for GSW epochs; Second row: BOLD-perfusion correlation for rest epochs; Third row: BOLD-perfusion correlation for entire time series. Fourth row: ASL-B SPM $\{t\}$ for negative weighting on HRF. Fifth row: Statistics map showing regions exhibiting a significant correlation from the ANCOVA analysis plotted with regions of significant BOLD deactivation. Dark blue indicates regions that were neither deactivated nor showed BOLD-perfusion correlation. Mid blue regions show a significant positive correlation between BOLD and perfusion with light blue indicating areas that showed a significant difference in correlation between GSW and rest. Orange and red colors demonstrate co-localisation of BOLD deactivation and correlation.

A strong positive correlation (yellow and red colours in row 3) is seen throughout the cortex during GSW. Regions of BOLD deactivation overlapped with significant correlation apart from at the very edges of the brain. The highest correlation is in the posterior parietal, posterior cingulate and frontal areas, with a significant difference in correlation between rest and GSW epochs within some of these areas (dark red in statistics maps). The orange/red regions in the statistics maps show that NBR was always associated with decreased perfusion.

b) BOLD-perfusion correlation maps and ASL-B SPM $\{t\}$ for all patients (representative slice) - analogous figure to a), but now per subject instead of per slice. Top row: BOLD-perfusion correlation for GSW epochs; Second row: BOLD-perfusion correlation for rest epochs; Third row: BOLD-perfusion for entire time series. Fourth 
row: ASL-B SPM $\{\mathrm{t}\}$ for negative weighting on HRF; Fifth row: Statistics map showing regions exhibiting a significant correlation from the ANCOVA analysis overlaid with regions of significant BOLD deactivation. Dark blue indicates regions that were neither deactivated nor showed BOLD-perfusion correlation. Mid blue regions show a significant positive correlation between BOLD and Perfusion with light blue indicating areas that showed a significant change in correlation between GSW and rest. Orange and red colors demonstrate co-localisation of BOLD deactivation and correlation with perfusion; sixth row: Statistics maps, as above, showing regions exhibiting a significant correlation from the ANCOVA analysis, only here, plotted with regions of significant BOLD activation for each subject. A positive correlation was always seen between BOLD and perfusion time courses in regions with either a significant BOLD activation or deactivation. The strongest correlations were found in the frontal, posterior parietal and posterior cingulate cortex. Stronger correlations found during GSW occur in regions of highest correlation during GSW or lowest correlation at rest and are likely to reflect the larger signal changes relative to noise in both the perfusion and BOLD voxel time course in these areas. 
Figure 1

\section{Patient (1) SGE}

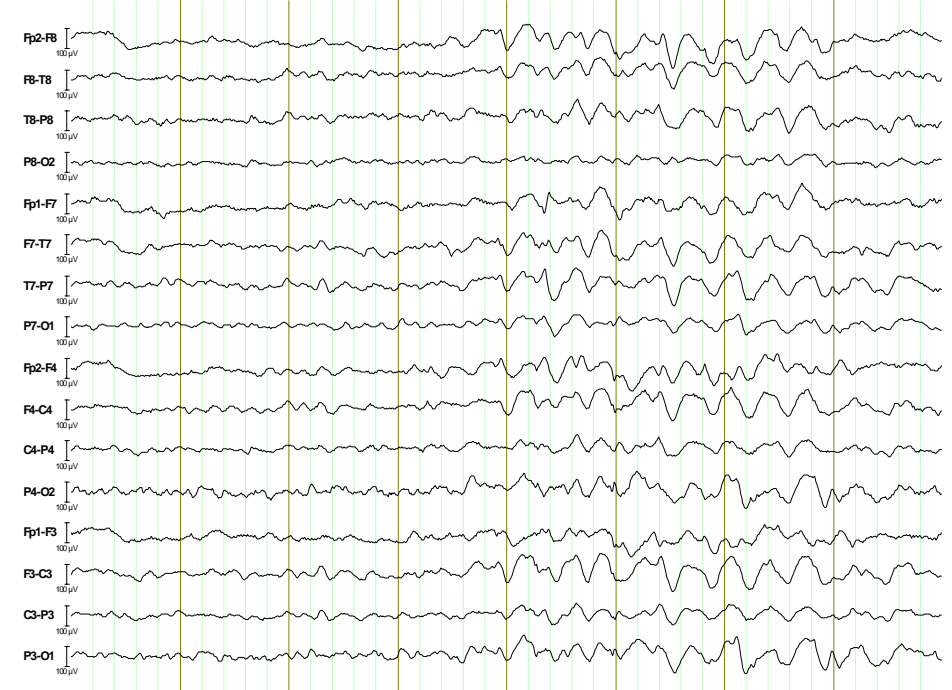

\section{Patient (3) EGTCS}

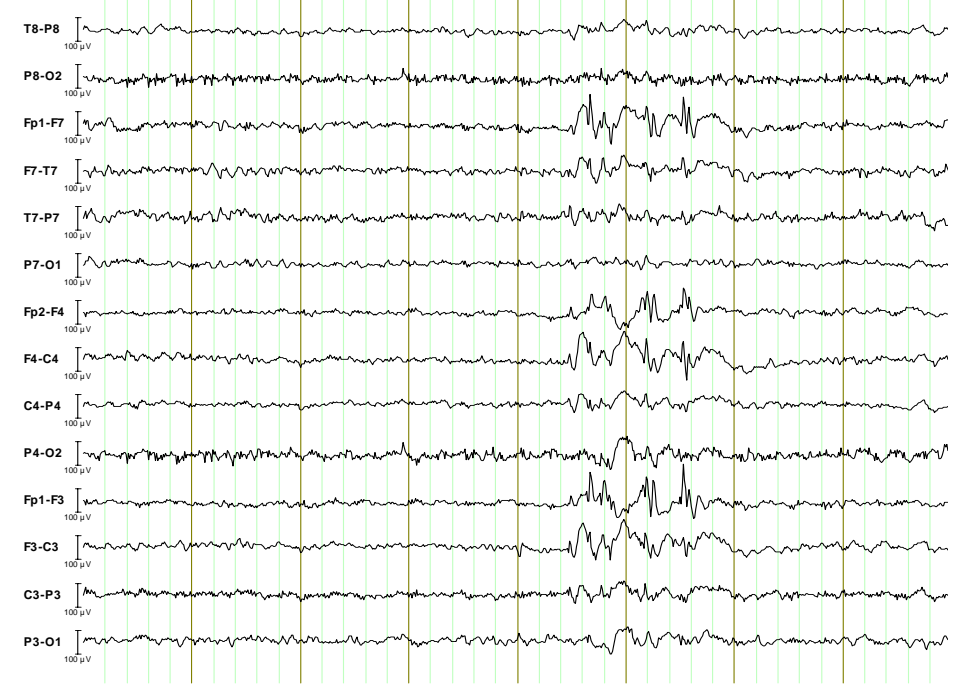

\section{Patient (2) SGE}

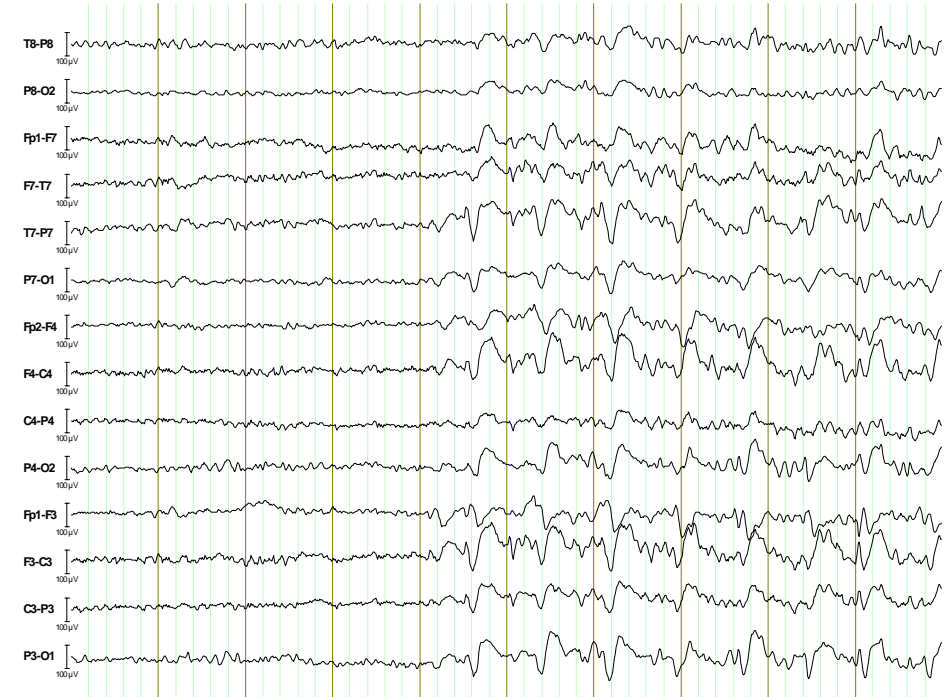

\section{Patient (4) CAE}

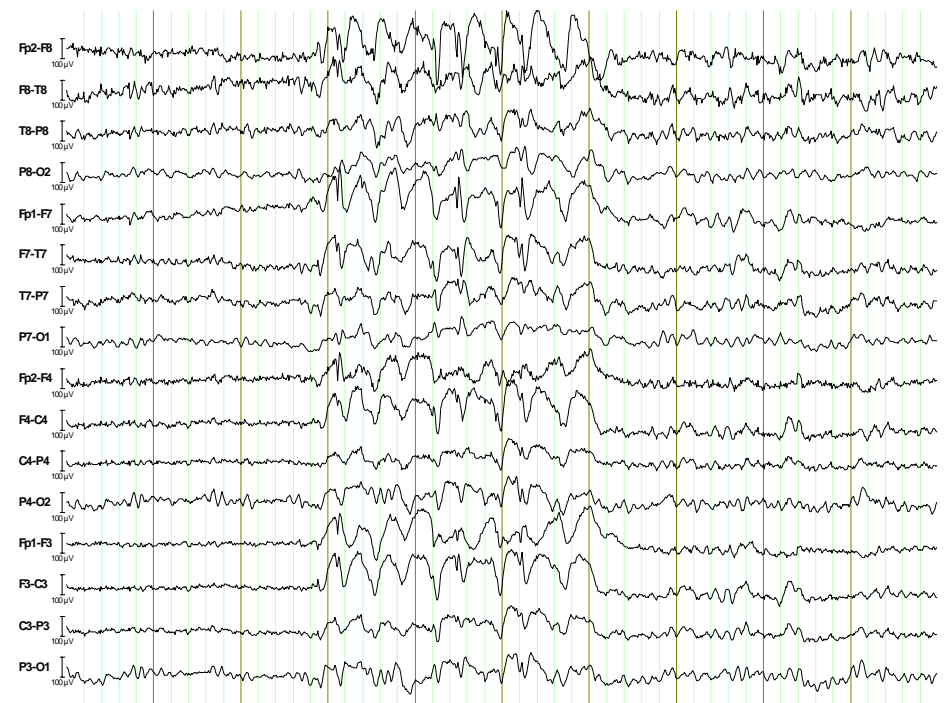


Patient (1) SGE
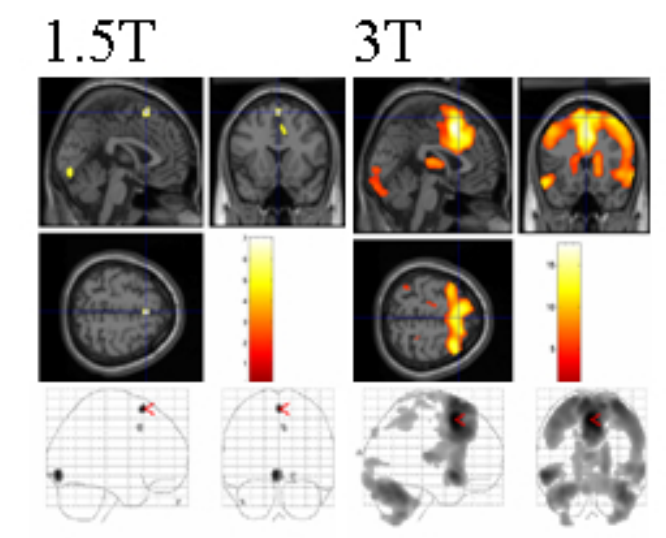

$-\mathrm{Cl}^{-2}$
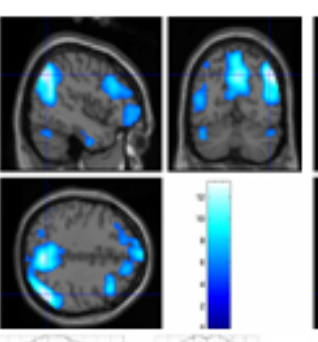

(5) है

$6)^{m-1}$

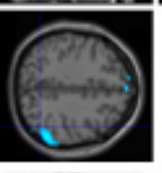

+4. 5 is

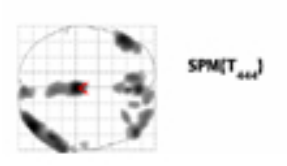

Patient (2) SGE
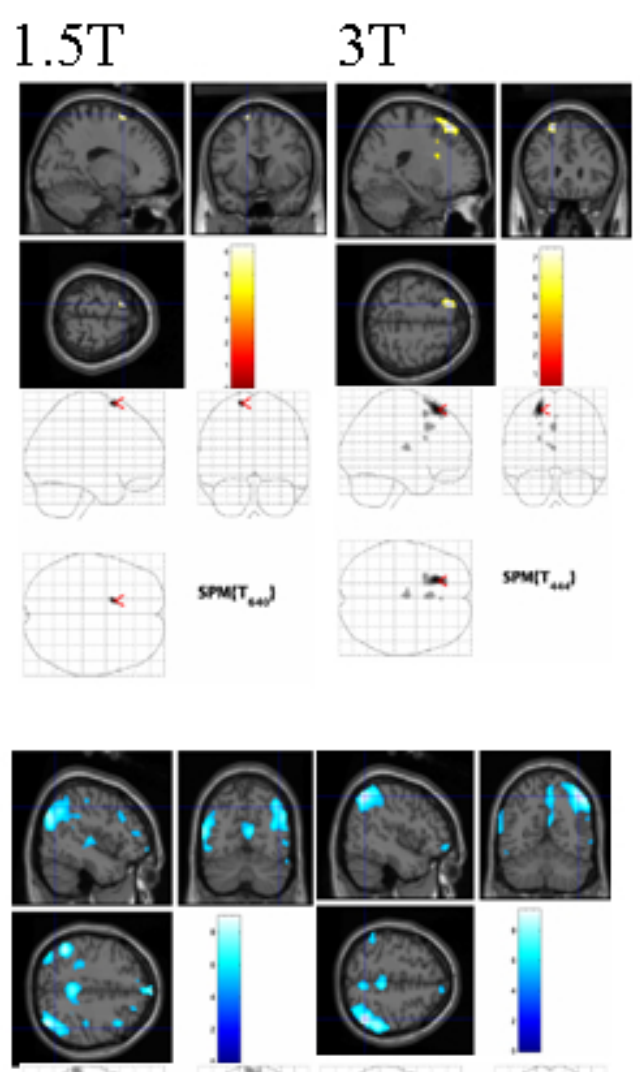

Patient (3) EGTCS

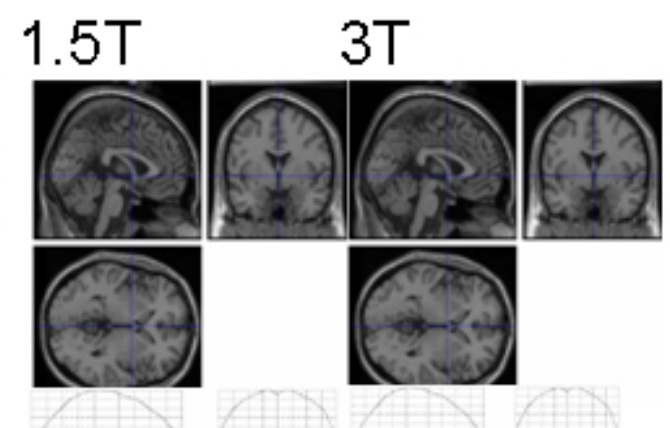

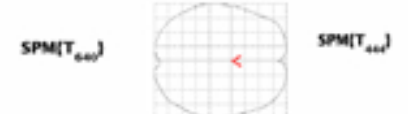
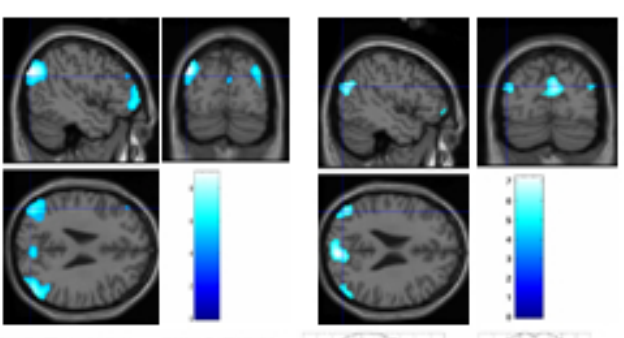

$8,100+2 \div$

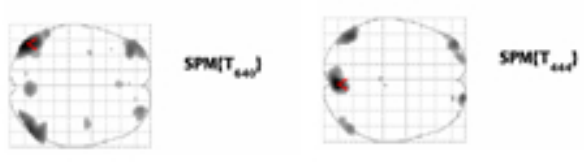

Patient (4) CAE

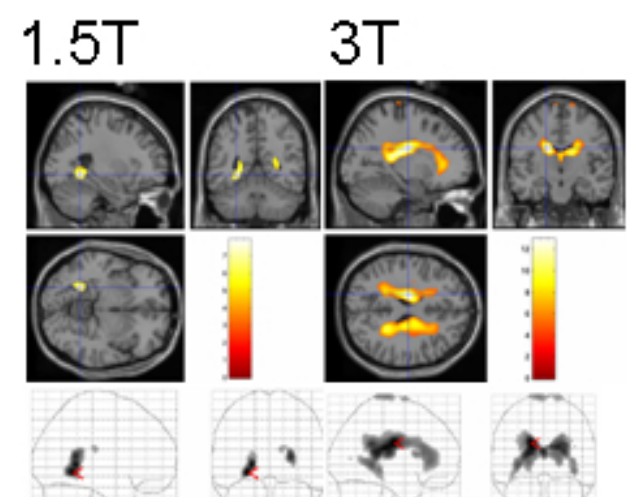

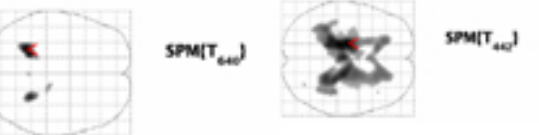

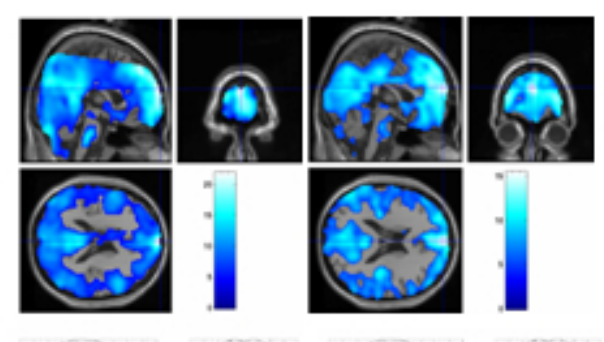

(3) 96

$67^{\cdots}$ 


\section{Patient (1) SGE}

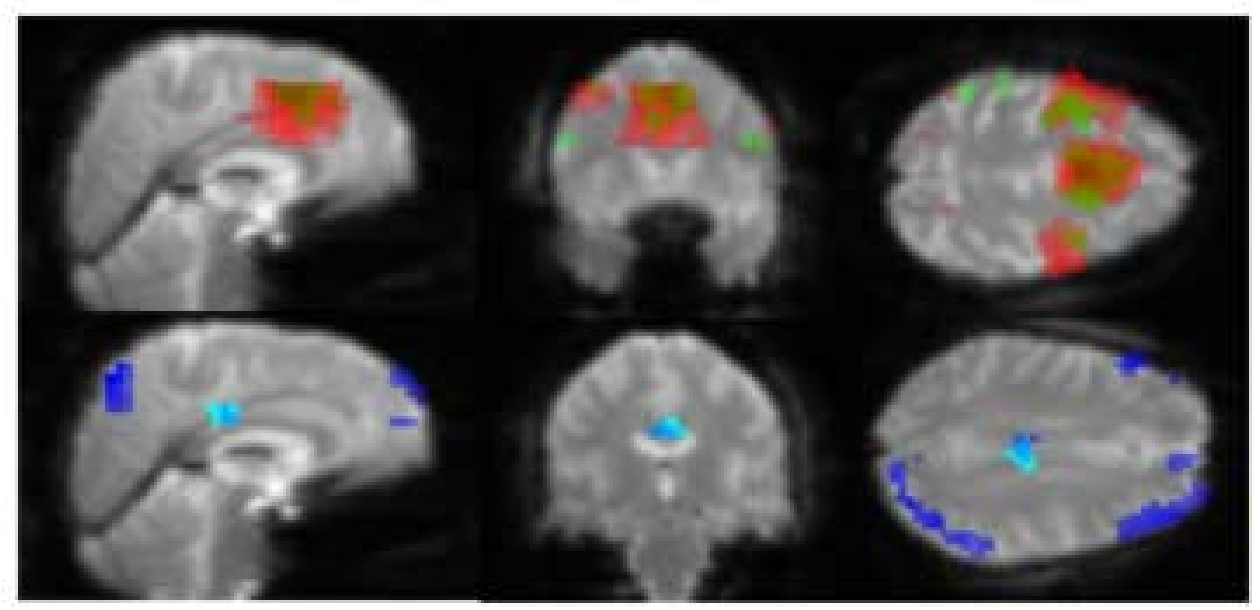

\section{Patient (2) SGE}

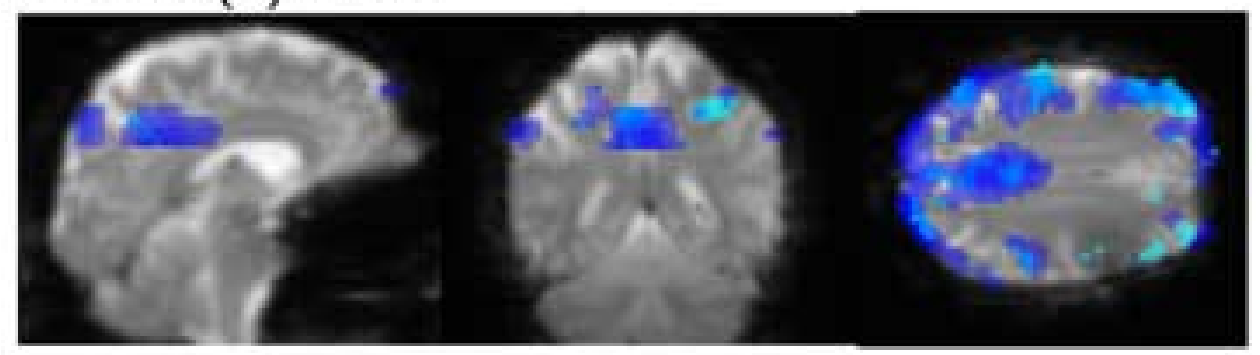

\section{Patient (4) CAE}

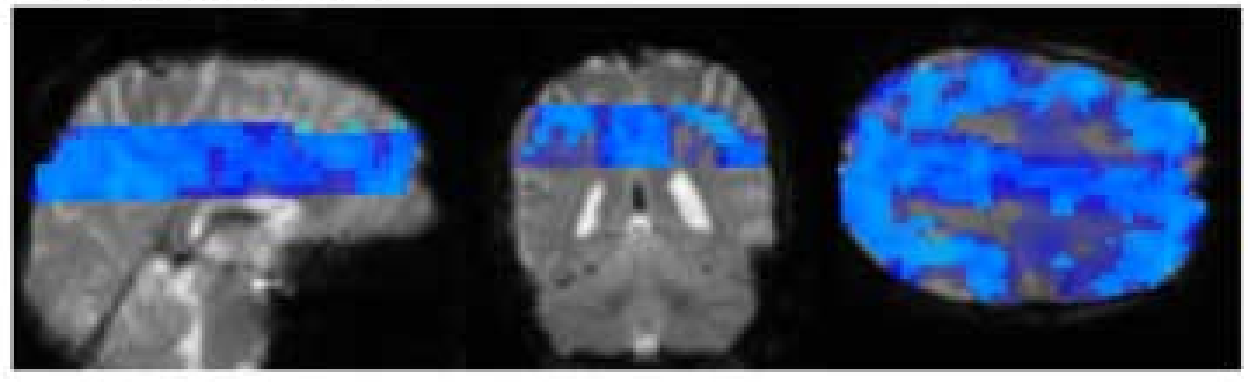




\section{Figure $4 a$}

Click here to download high resolution image

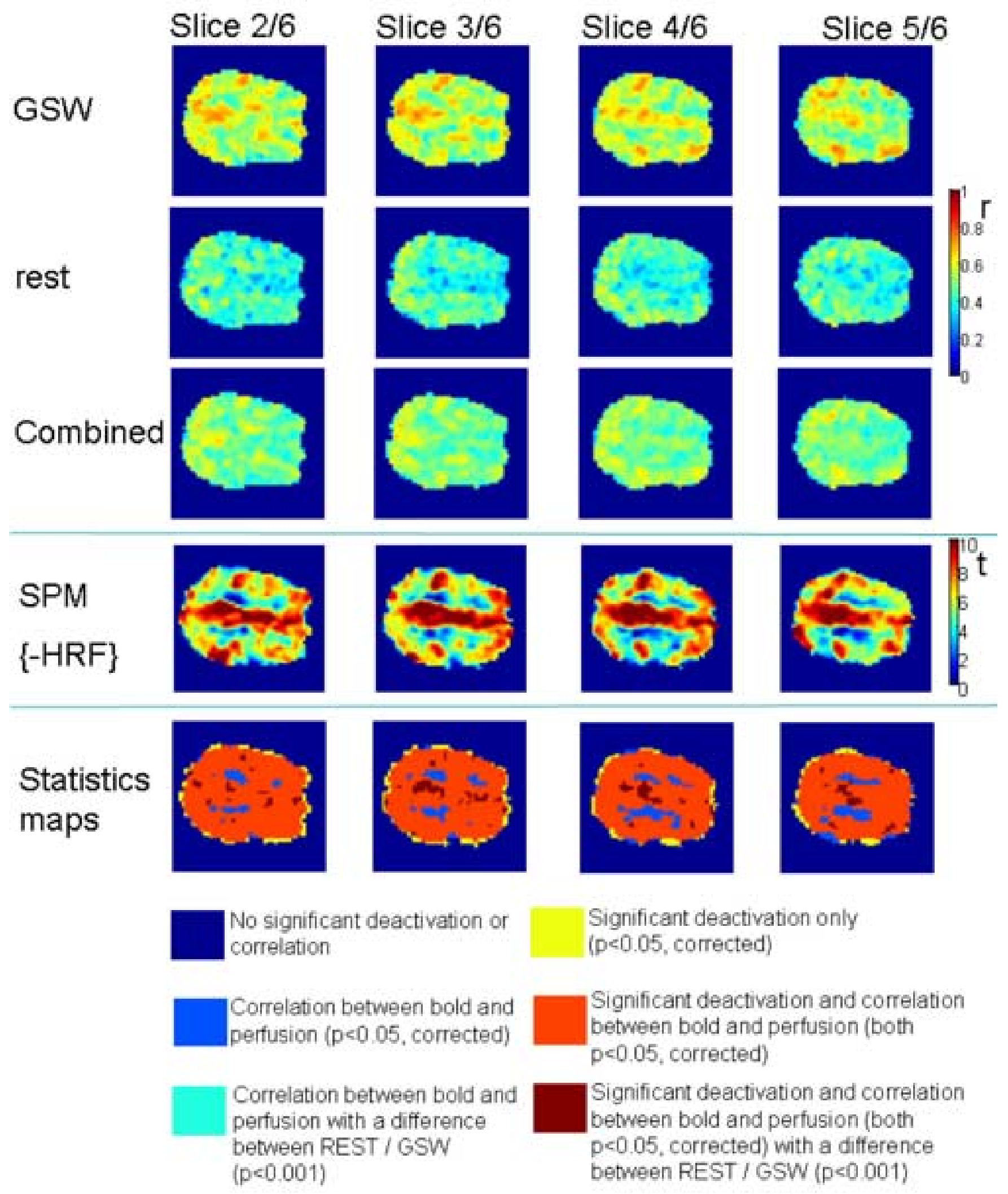




\section{Figure $4 b$}

Click here to download high resolution image

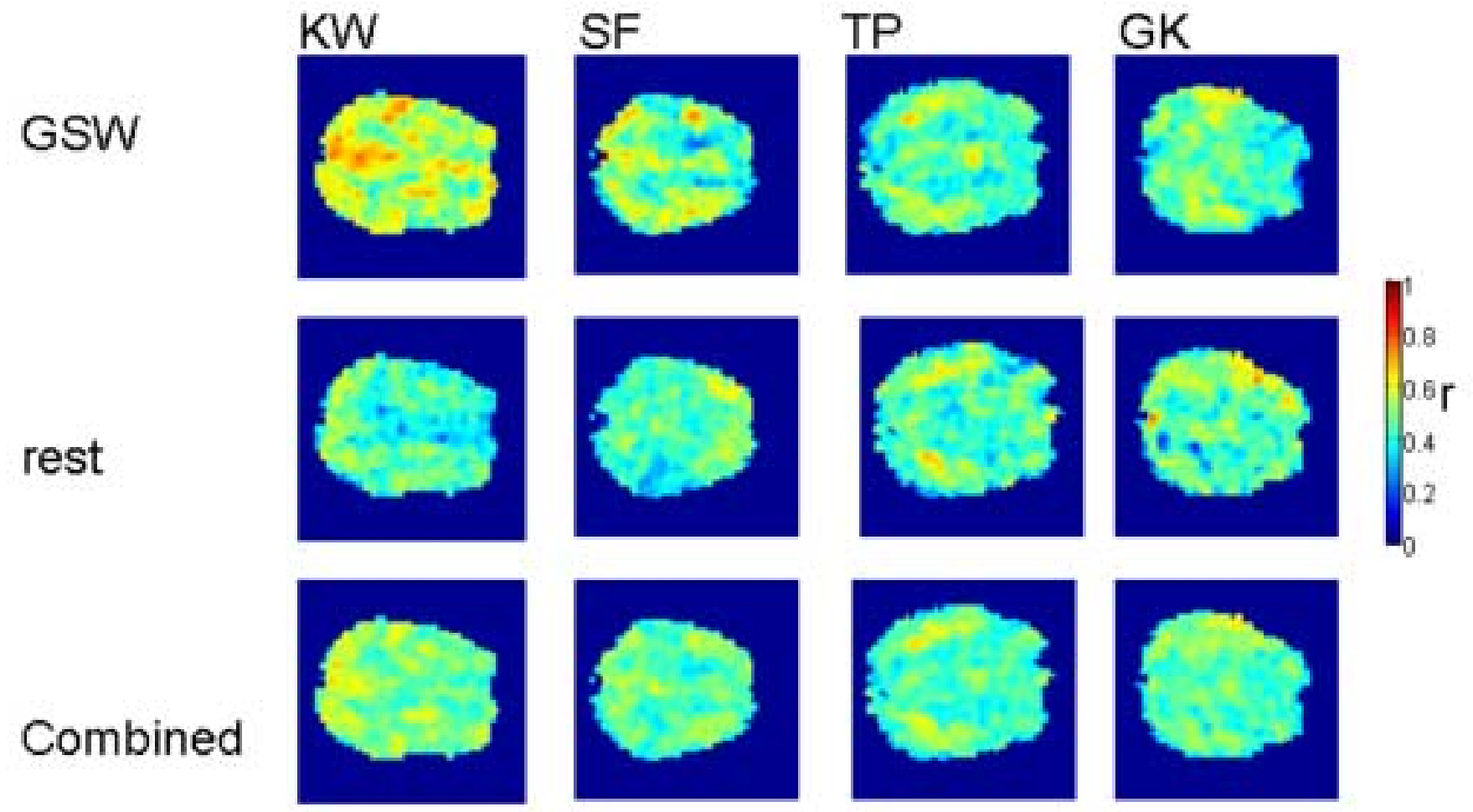

Statistics

maps with

BOLD-ASL

deactivation
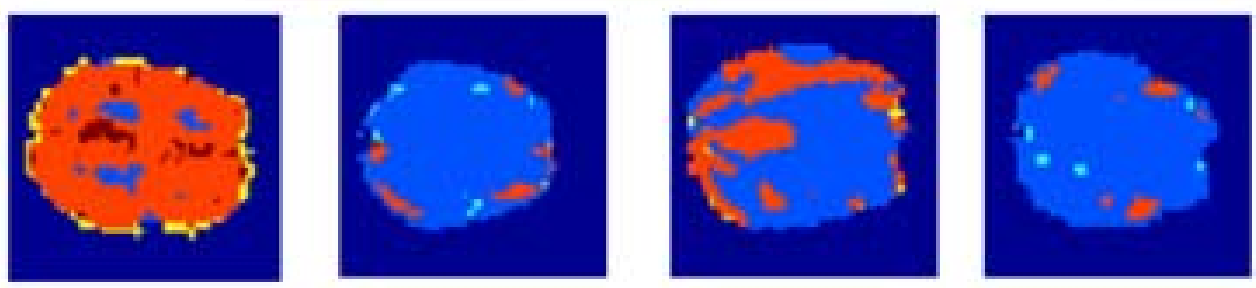

Statistics

maps with BOLD-ASL activation
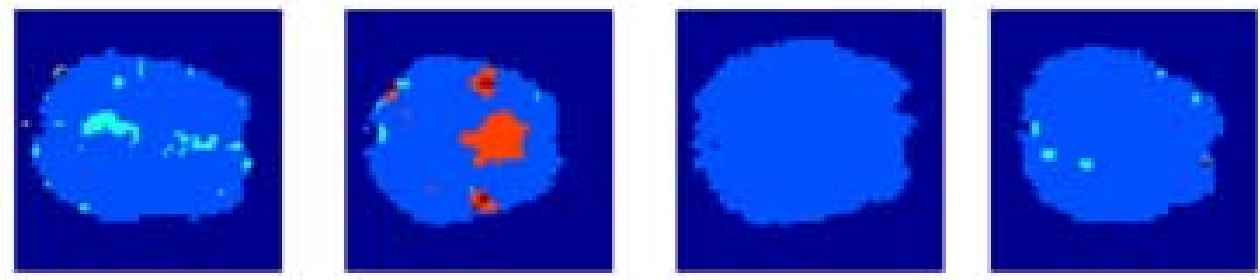

No significant (de/)activation or correlation

Significant (de/)activation only ( $<<0.05$, corrected)

Correlation between bold and perfusion ( $p<0.05$, corrected)

Correlation between bold and perfusion with a difference between REST / GSW $(p<0.001)$

Significant (de/)activation and correlation between bold and perfusion (both p<0.05, corrected)

Significant (de/)activation and correlation between bold and perfusion (both $p<0.05$, corrected) with a difference between REST / GSW (p<0.001) 


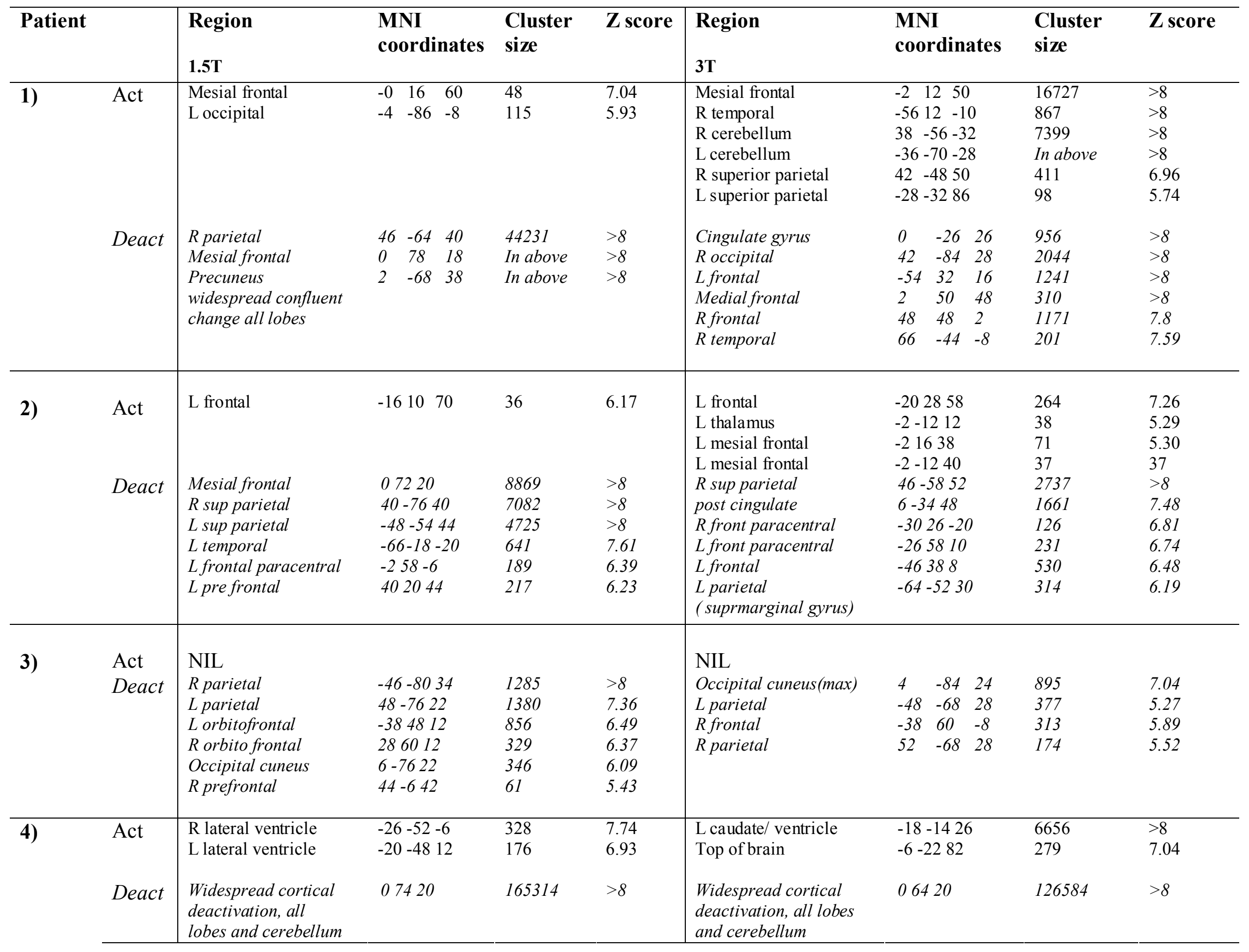




\section{Supplementary material}

Table 2. Details of main local maxima from SPM $\{t\}$ of positive and negative HRF for the 1.5 and $3 T$ BOLD fMRI sessions for each patient. $1.5 \mathrm{~T}$ and $3 \mathrm{~T}$ are shown side by side for comparison. The positive weighting on the HRF is denoted Act (activation), the negative Deact (deactivation), shown in italics. MNI coordinates, Z score and cluster size are given in each case. 